\title{
Sensorless Implementation of a PPF Controller for Active $Q$ Control of an AFM Microcantilever
}

\author{
Matthew W. Fairbairn, Member, IEEE, Philipp Müller, and S. O. Reza Moheimani, Fellow, IEEE
}

\begin{abstract}
Reducing the cantilever quality $(Q)$ factor in the atomic force microscope (AFM), when operating in tapping mode, allows for an increase in imaging speed. Passive piezoelectric shunt control has several advantages over alternative methods of cantilever $Q$ factor reduction. However, this technique uses a passive electrical impedance to modify the mechanical dynamics of the cantilever, which limits the amount of $Q$ factor reduction achievable. This paper demonstrates that further reductions in the cantilever $Q$ factor may be obtained with the use of an active impedance in the piezoelectric shunt control framework. The active impedance parameters are designed in such a way that the piezoelectric shunt controller emulates a positive position feedback controller in a displacement feedback control loop. A significant reduction in cantilever $Q$ factor is obtained using an active impedance compared with that achieved with a passive impedance. The improvement in scan speed using this control technique is demonstrated with AFM images of a test sample.
\end{abstract}

Index Terms-Atomic force microscope (AFM), AFM probe, microcantilevers, microsensors, piezoelectric cantilever, piezoelectric shunt control, synthetic impedance, tapping-mode AFM.

\section{INTRODUCTION}

$\mathbf{T}$ HE atomic force microscope (AFM) [1] is one of the leading instruments for high resolution imaging of samples with nanoscale features. Tapping mode [2]-[4] is the most prevalent AFM imaging mode, as sample damage and distortion are greatly reduced compared with alternative modes of operation.

When imaging in tapping mode, the sample is interrogated by lightly tapping the surface with a sharp probe tip, which is located on the underside of a microcantilever. See Fig. 1 for a schematic of a typical AFM operating in tapping mode. The probe tip is oscillated at, or close to, the cantilever's first resonance frequency while the sample is scanned beneath the cantilever, typically in a raster pattern, using a piezoelectric scanner. A 3-D image of the sample topography is generated by recording the output of the $z$-axis feedback controller, which maintains the cantilever oscillation amplitude at a setpoint value by raising or lowering the height of the sample stage.

Manuscript received July 3, 2013; revised October 24, 2013; accepted January 6, 2014. Manuscript received in final form January 27, 2014. Date of publication February 19, 2014; date of current version October 15, 2014. Recommended by Associate Editor I. Petersen.

M. W. Fairbairn and S. O. R. Moheimani are with the School of Electrical Engineering and Computer Science, University of Newcastle, Callaghan, NSW 2308, Australia (e-mail: matthew.fairbairn@newcastle.edu.au; reza.moheimani@newcastle.edu.au).

P. Müller is with the Institute for Systems Theory and Automatic Control, University of Stuttgart, Stuttgart 70174, Germany (e-mail: mueller.philipp@ rocketmail.com).

Color versions of one or more of the figures in this paper are available online at http://ieeexplore.iee.org.

Digital Object Identifier 10.1109/TCST.2014.2303486

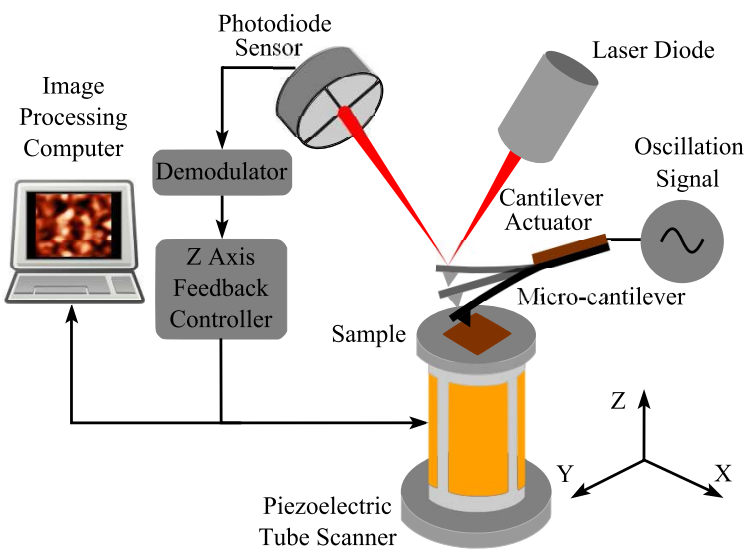

Fig. 1. Instrumentation of a typical AFM operating in tapping mode.

In many imaging applications, high scan speeds are desirable to increase productivity [5] and capture dynamic processes [6], [7]. Tapping mode AFM has a relatively slow scan speed compared with alternative imaging modes. The maximum obtainable scan speed is limited by the bandwidth of the $z$-axis feedback loop, which is inversely proportional to the quality $(Q)$ factor of the sensing cantilever [8]-[10]. Significant increases in scan speed have been demonstrated with the use of active $Q$ control to reduce the cantilever $Q$ factor [8]-[11].

Direct velocity feedback may be used to modify the effective cantilever $Q$ factor as the damping force is proportional to the cantilever tip velocity. The compact size of the AFM and the presence of the existing displacement sensor would make installation of a separate velocity sensor impractical. Active $Q$ control is therefore typically implemented by estimating the tip velocity from the cantilever displacement signal. To avoid amplification of high frequency noise in the feedback-loop differentiation of the displacement signal is not recommended.

As the cantilever displacement is sinusoidal, delaying the signal by $\pi / 2 \mathrm{rad}$, with a time delay circuit, provides an estimate of the tip velocity. This is the most common implementation of active $Q$ control in commercial AFMs.

The time delay controller may have a spill-over effect [12] on higher order resonance modes of the cantilever, which may lead to a reduction in scan speed rather than an increase, and in some cases result in instability of the cantilever [10], [13]. For this reason, researchers have developed other control techniques to modify the cantilever $Q$ factor such as observer-based control [14], resonant control [10], and piezoelectric shunt control [11], [15]. 


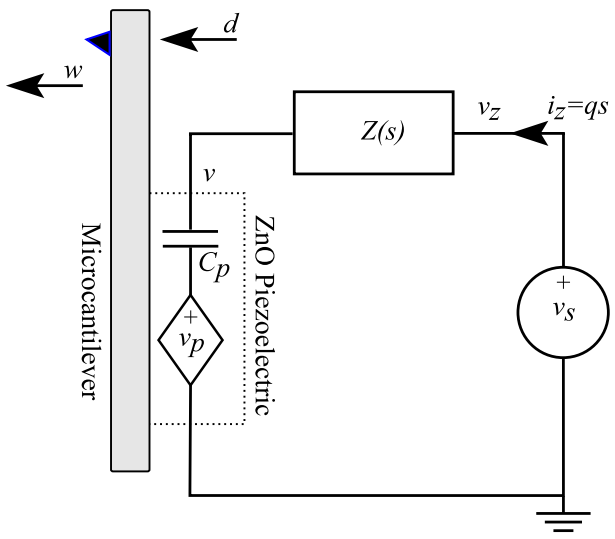

Fig. 2. Self actuated piezoelectric AFM microcantilever with attached shunt circuit.

Passive piezoelectric shunt control has an advantage over both observer-based control and resonant control in that the optical sensor [16], typically used to measure cantilever deflection, is removed from the active $Q$ control feedback loop. The optical sensor introduces a significant amount of measurement noise into the feedback loop [17]. Therefore, elimination of this sensor from the feedback loop is an advantage. In [11], we demonstrated the advantages of using passive piezoelectric shunt control to decrease the cantilever $Q$ factor for increased scan speeds when imaging in air. There is a limitation to how far the cantilever $Q$ factor may be reduced using this technique due to the passive nature of the shunt impedance used. Further reductions in the cantilever $Q$ factor may be desired for increased scan speeds or for such applications where it is desirable to damp the first mode as much as possible to operate on the second cantilever mode [18]. This paper presents an extension of the piezoelectric shunt control technique, which uses an active impedance to obtain a further reduction in the cantilever $Q$ factor. The parameters of the shunt impedance are designed in such a way that the closedloop system emulates a displacement feedback loop with a positive position feedback (PPF) [19] controller.

\section{Piezoelectric Shunt Control}

The piezoelectric shunt control technique makes use of a self actuated piezoelectric microcantilever, which has a layer of piezoelectric material attached to the cantilever beam. A sinusoidal voltage is applied to this piezoelectric actuator to oscillate the cantilever when operating in tapping mode. When the piezoelectric material is subjected to a strain a voltage $v_{p}$ is generated according to the relationship $\alpha=v_{p} / d$, where $\alpha$ is the actuator voltage to displacement coefficient and $d$ is the cantilever tip displacement. This conversion of mechanical energy to electrical energy is the basis of piezoelectric shunt control. By placing an electrical impedance in series with the oscillation voltage source $v_{s}$ and tuning the dynamics of the resulting electrical circuit to the mechanical dynamics of the cantilever, the cantilever $Q$ factor may be modified.

A schematic diagram of the piezoelectric shunt control system is shown in Fig. 2, where the piezoelectric material

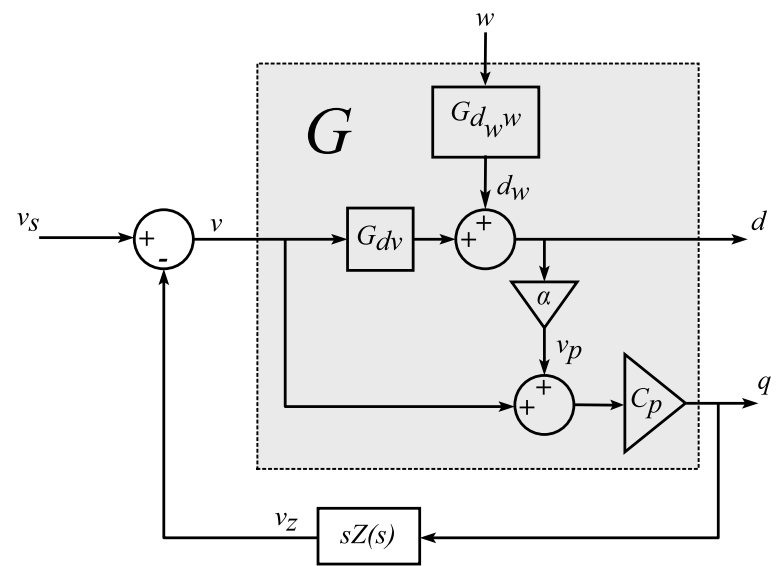

Fig. 3. Block diagram of the piezoelectric shunt controlled system.

attached to the cantilever beam is modeled as a capacitance $C_{p}$ in series with a strain-dependent voltage source $v_{p}$ [20].

The piezoelectric shunt control system may be represented by the block diagram shown in Fig. 3 where the cantilever is modeled as the system $G(s)$ with inputs $w$ (a disturbance strain on the cantilever) and $v$ (the voltage across the piezoelectric transducer terminals), and outputs $d$ (cantilever tip displacement) and $q$ (charge generated by the piezoelectric transducer). In this representation, $v_{z}$ is the voltage across the shunt impedance and $d_{w}$ is the initial tip displacement due to a sample perturbation. The transfer function from $v$ to $d$ is modeled as

$$
G_{d v}(s)=\frac{d(s)}{v(s)}=\frac{\beta_{v} \omega_{n}^{2}}{s^{2}+\frac{\omega_{n}}{Q} s+\omega_{n}^{2}}
$$

and the transfer function from $w$ to $d_{w}$ is is modeled as

$$
G_{d_{w} w}(s)=\frac{d_{w}(s)}{w(s)}=\frac{\beta_{w} \omega_{n}^{2}}{s^{2}+\frac{\omega_{n}}{Q} s+\omega_{n}^{2}}
$$

where $\omega_{n}$ is the natural frequency of the cantilever, and $\beta_{v}$ and $\beta_{w}$ are the steady-state gains of $G_{d v}(s)$ and $G_{d_{w} w}(s)$, respectively.

By representing the piezoelectric shunt control system in block diagram form, as shown in Fig. 3, the transfer function from the applied voltage $v_{s}$ to tip displacement $d$ is determined to be [11]

$$
G_{d v_{s}}(s)=\frac{H(s) G_{d v}(s)}{1+K(s) G_{d v}(s)}
$$

where

$$
K(s)=\frac{s Z(s) C_{p} \alpha}{1+s Z(s) C_{p}}
$$

and

$$
H(s)=\frac{1}{1+s Z(s) C_{p}} .
$$

In this form, $G_{d v_{s}}(s)$ is equivalent to a displacement negative feedback loop with a controller $K(s)$ and a prefilter $H(s)$, as shown in Fig. 4. The prefiltering effect is due to the electrical dynamics of the shunt circuit. 


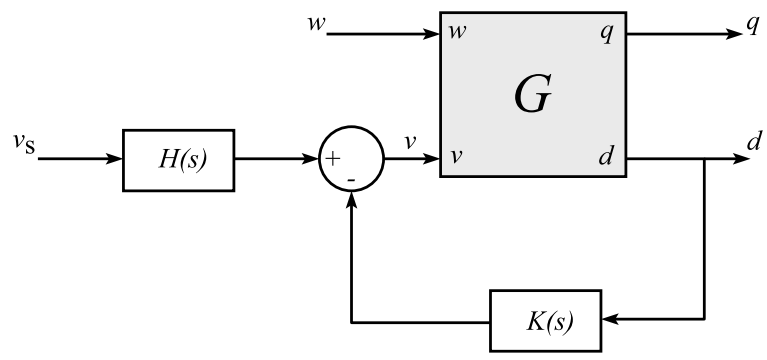

Fig. 4. Equivalent displacement feedback system from $v_{s}$ to $d$ and from $w$ to $d$.

The prefiltering of the sinusoidal input signal $v_{s}$ modifies the magnitude and phase of the signal applied to the system $G(s)$. The phase delay due to $H(s)$ does not affect the operation of the device and the change in magnitude may be compensated for by adjusting the magnitude of $v_{s}$. The poles of the transfer function from a disturbance, due to a change in sample topography, to the tip displacement determine the maximum scan speed at which the device may be operated. This transfer function was determined to be

$$
G_{d w}(s)=\frac{G_{d_{w} w}(s)}{1+K(s) G_{d v}(s)}
$$

which is not affected by the prefilter $H(s)$.

In [11], the passive impedance used to reduce the cantilever $Q$ factor consisted of an inductance $L$ in series with a resistance $R(Z(s)=L s+R)$. This shunt impedance resulted in an equivalent displacement negative feedback controller

$$
K(s)=\frac{\alpha s\left(s+\frac{R}{L}\right)}{s^{2}+\frac{R}{L} s+\frac{1}{C_{p} L}} .
$$

This is a resonant controller [21], which has a phase lead of $\pi / 2$ at the resonance frequency. If the controller resonance frequency is tuned to the oscillation frequency of the cantilever the controller is effectively estimating the cantilever tip velocity and applying a gain. The gain and bandwidth of control may be tuned by varying the parameters $R$ and $L$.

The passive piezoelectric shunt control technique has a limitation in the amount of damping attainable due to limitations of the transducer and the passive nature of the impedance used. A passive impedance cannot supply energy to the system and will therefore only use the electric energy supplied by the piezoelectric layer to modify the mechanical dynamics of the cantilever. If further reductions in the cantilever $Q$ factor are desired then it is necessary to design an active impedance [22].

\section{Emulating A Displacement FeEdBack PPF Controller With Active Piezoelectric SHUNT CONTROL}

From (4), it can be observed that for any displacement negative feedback controller there is an equivalent shunt impedance transfer function [23]. To obtain the equivalent shunt impedance, (4) must to be solved for Z(s), which results in

$$
Z(s)=\frac{K(s)}{s C_{p}(\alpha-K(s))}
$$

If the gain of the controller is high enough the resulting impedance will be active.

In this paper, the equivalent shunt impedance for a PPF controller is derived. The PPF controller is a second-order negative imaginary controller, defined as

$$
K(s)=\frac{a}{s^{2}+2 \zeta_{c} \omega_{c} s+\omega_{c}^{2}}
$$

where $a$ and $\zeta_{c}$ are the control design parameters, which determine the gain at the frequency of interest. To estimate the tip velocity at the cantilever oscillation frequency, $\omega_{c}$ should be set equal to $\omega_{n}$ and the parameter $a$ must be negative, to ensure that the controller has a phase lead of $\pi / 2$ at $\omega_{n} \cdot \zeta_{c}$ is set much higher than the damping factor of the system to be controlled.

The PPF controller was chosen for this application as the controller transfer function rolls off at a rate of $40 \mathrm{~dB} /$ decade for high frequencies. This makes the closed-loop system robust to spillover effects on unmodeled high frequency cantilever modes and reduces noise in the feedback loop.

For the PPF controller, the equivalent impedance obtained by substituting (9) in (8) is

$$
Z_{\mathrm{PPF}}(s)=\frac{1}{s} \cdot \frac{a}{\alpha C_{p} s^{2}+2 \alpha C_{p} \zeta_{c} \omega_{c} s+\alpha C_{p} \omega_{c}^{2}-a C_{p}} .
$$

\section{A. Passivity of the PPF Controller Equivalent Impedance}

An electric impedance is passive when it does not deliver any power to the system. This can be expressed as [24], [25]

$$
\int_{0}^{\infty} v_{z}(t) \cdot i_{z}(t) d t \geq 0 .
$$

For a linear impedance $Z(s),(11)$ is fulfilled when $Z(s)$ is positive-real. A linear transfer function is positive real if and only if [24].

1) $Z(s)$ has no poles in right half plane.

2) The poles on the $j \omega$ axis are simple and their residues are real and positive.

3) $\Re[Z(j \omega)] \geq 0 \forall \omega$.

When the parameter $a$ is negative and all other parameters are positive, $Z_{\mathrm{PPF}}(s)$ meets all of the above conditions except condition 2 . The residuum of the one pole on the $j \omega$-axis of $Z_{\mathrm{PPF}}(s)$ is equal to

$$
\frac{a}{\alpha C_{p} \omega_{c}^{2}-a C_{p}}
$$

which is negative when $a$ is negative. This proves that $Z_{\mathrm{PPF}}(s)$ is an active impedance.

\section{Comparison of the Damping Performance of Active and Passive Piezoelectric SHUNT CONTROL}

In this section, the parameters of a passive piezoelectric shunt controller are designed to provide the maximum amount of damping achievable for a particular cantilever. An active impedance is then designed in the piezoelectric shunt control framework, which produces significantly higher damping than that obtained with the passive impedance. 


\section{A. Identification of Cantilever Parameters}

The Dimension Micro-actuated Silicon Probe (DMASP) AFM microcantilever, available from Bruker AFM Probes [26], was used in this paper. The parameters of the DMASP microcantilever were measured to obtain a model of the cantilever, which was used to compare the damping obtainable with the passive and active piezoelectric shunt controllers.

A frequency response of $G_{d v}(s)$ was obtained using a microscope scanning laser Doppler vibrometer (Polytec MSV 400). The cantilever was excited by applying a pseudorandom signal, and the resulting tip displacement measured. A mathematical model of $G_{d v}(s)$ was then obtained by system identification and is given by

$$
G_{d v}(s)=\frac{2550}{s^{2}+1600 s+1.3005 \times 10^{11}} .
$$

The resonance frequency $\left(f_{r}\right)$ was measured from the frequency response to be $57406 \mathrm{~Hz}$.

$\alpha$ and $C_{p}$ are the properties of the cantilever, which must be determined to design $K(s)$. $C_{p}$ was measured at $24 \mathrm{pF}$ using an Agilent E4980A LCR meter. The value of $\alpha$ was determined by measuring the frequency response of the admittance $G_{i v}(s)$. The frequency response of $G_{i v}(s)$ was obtained by applying a swept sine current to the cantilever and measuring the resulting voltage appearing across its electrodes. The model of $G_{i v}(s)$, obtained from the frequency response through system identification, was determined to be

$$
G_{i v}(s)=\frac{2.395 \times 10^{-11} s^{3}+3.831 \times 10^{8} s^{2}+3.115 s}{s^{2}+1600 s+1.301 \times 10^{11}} .
$$

The admittance transfer function is given by [11]

$$
G_{i v}(s)=\frac{i(s)}{v(s)}=\alpha C_{p} G_{d v}(s) s+C_{p} s .
$$

By equating (14) to (15) $\alpha$ was determined to be $\approx 2 \times 10^{4}$.

\section{B. Tuning of the Passive Shunt Controller}

The cantilever model was then used to determine the shunt impedance parameters, which will result in the maximum reduction in the cantilever $Q$ factor obtainable when using a passive impedance $Z(s)=L s+R$. The parameters $L$ and $R$ were determined using the procedure described in [11].

The inductance was chosen to satisfy the relationship

$$
2 \pi f_{r}=1 / \sqrt{L C_{p}} .
$$

This means that the electrical resonance is at the same frequency as the mechanical resonance to ensure maximum interaction between the mechanical and electrical dynamics. Substituting the values for $f_{r}$ and $C_{p}$ into (16) gives a value of $L=0.321 \mathrm{H}$. To determine the value of $R$ the $H_{2}$ norm of the transfer function $G_{d w}(s)$ for a varying resistance was obtained in MATLAB. The $\mathrm{H}_{2}$ norm of a transfer function is proportional to the area under the frequency response magnitude curve. Reducing the area under this curve means that the damping of the system is increased. The resistance value, which produced the minimum $\mathrm{H}_{2}$ norm, and therefore the maximum reduction in $Q$ factor is $2330 \Omega$. The position of the closed-loop poles of the system were determined in

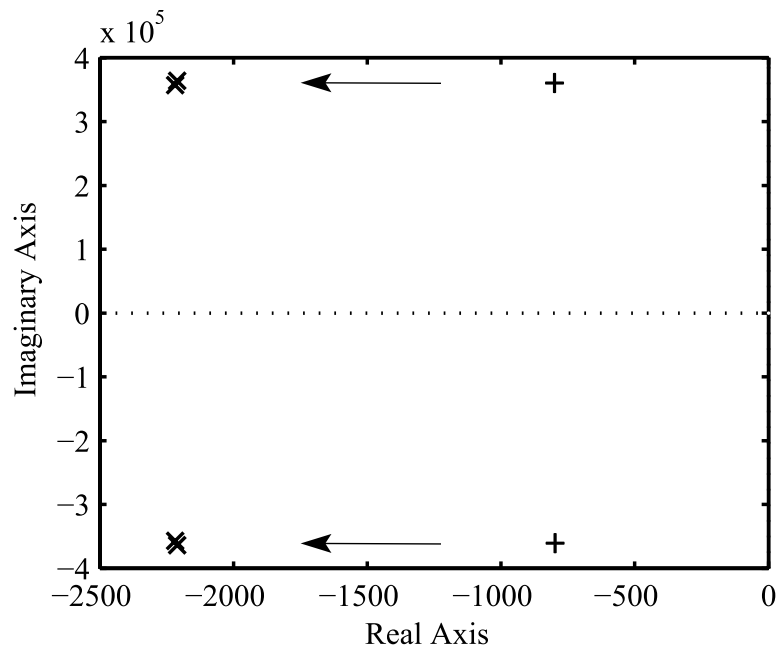

Fig. 5. Open (+), and closed-loop (x) pole locations of the cantilever using passive piezoelectric shunt control. The impedance used was $0.321 s+$ $2330 \Omega$.

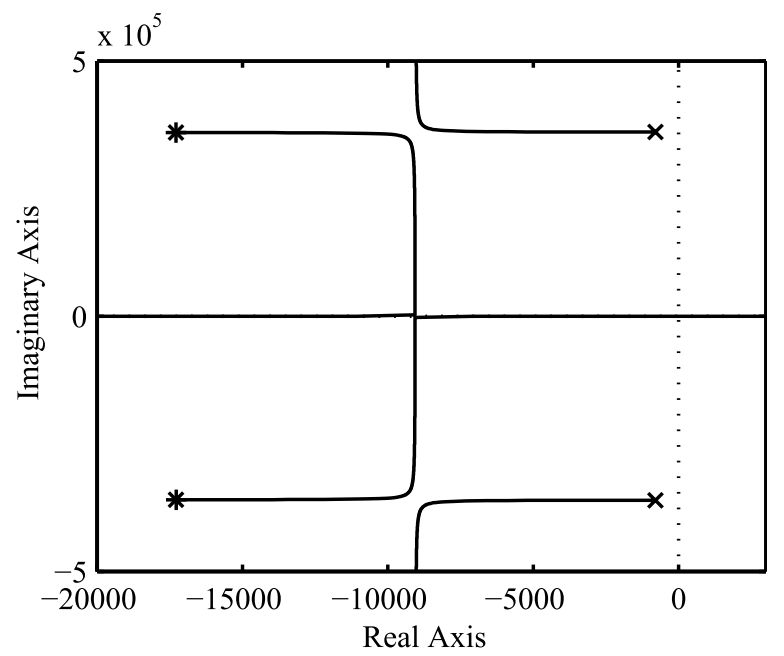

Fig. 6. Root locus of the cantilever when an active shunt impedance $Z_{\mathrm{PPF}}(s)$ is applied to implement the PPF controller $K(s)$. The fixed parameters were $\zeta_{c}=0.048$ and $\omega_{c}=360690 \mathrm{rad} / \mathrm{s} . \quad a$ is the variable parameter. $a \in[-\infty, 0]$. The open-loop cantilever poles $(+)$ and the poles of the PPF controller $(*)$ are shown in the plot.

MATLAB and shown in Fig. 5. The poles of $G_{d v}(s)$ with no shunt control are also shown in Fig. 5.

\section{Tuning of the PPF Controller for Implementation as an Active Shunt Controller}

The PPF controller has three parameters to tune $\omega_{c}, \zeta_{c}$ and $a$. For the PPF controller to approximate a differentiator at the cantilever oscillation frequency $\omega_{n}, \omega_{c}$ is set to be equal to $\omega_{n}$.

The closed-loop transfer function has four poles. When $\omega_{c}$ is equal to $\omega_{n}$ and $\zeta_{c}$ is set to a fixed value the root locus, with $a$ being the variable parameter, is plotted as shown in Fig. 6 . As $a$ is varied from $-\infty$ to 0 the closed-loop poles converge from the poles of $G_{d v}(s)$ and $K(s)$. As these poles move closer together they then split toward the open-loop zeros at $\infty$. For the closed-loop transfer function to have a frequency response, 


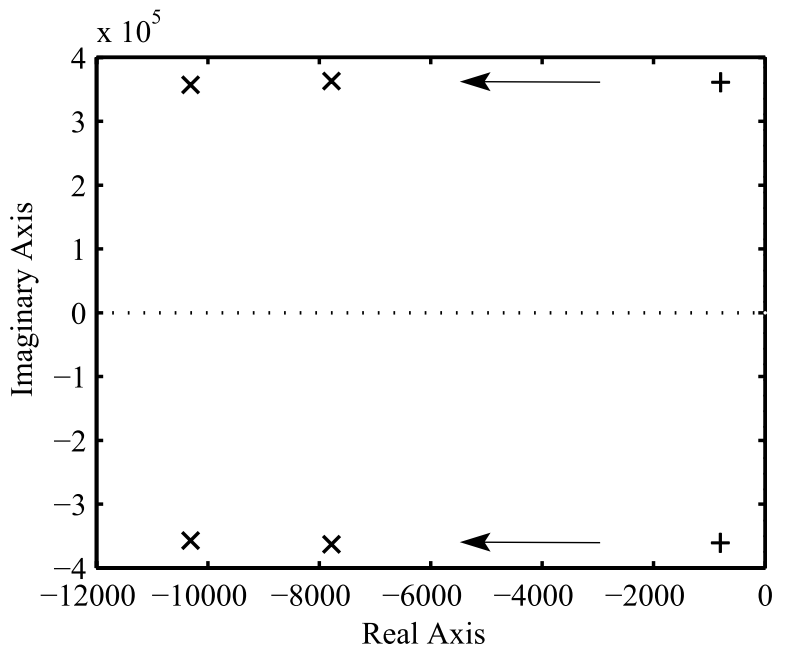

Fig. 7. Open- $(+)$ and closed-loop (x) pole locations of the cantilever when an active shunt impedance $Z_{\mathrm{PPF}}(s)$ is applied to emulate the PPF controller $K(s)$. The control parameters for $K(s)$ are $a=-1.2400 \times 10^{16}, \zeta_{c}=0.048$ and $\omega_{c}=360690 \mathrm{rad} / \mathrm{s}$.

which resembles a second-order system the four closed-loop poles must have the same magnitude in the imaginary axis of the complex plane. For the DMASP cantilever used in these experiments $\omega_{n}=2 \pi \times 57406 \mathrm{rad} / \mathrm{s}$. From (13), the damping coefficient of the cantilever is determined to be $\zeta=0.0022$. A value of $\zeta_{c}=0.048$ was chosen to place the poles of $K(s)$ deeper into the left half of the complex plane than the closed-loop poles obtained with passive piezoelectric shunt control shown in Fig. 5. This value was chosen experimentally as it allowed for the poles of the controller to be pushed significantly deeper into the left half of the complex plane while not being so large that an unrealizable gain $a$ would be required. Increasing $\zeta_{c}$ would make the controller more robust but it would require a much higher gain making the impedance difficult to implement. The root locus with these parameters, and with $a$ being the variable parameter, is shown in Fig. 6 . The value of $a$ for which the poles had converged and were about to split along the imaginary axis was chosen. This is the value of $a$, which will give the maximum reduction in cantilever $Q$ factor while ensuring that the closed-loop poles do not differ significantly in frequency. The value chosen for $a$ is $-1.2400 \times 10^{16}$.

From (10), the impedance which would be required to implement the above controller is

$$
Z_{\mathrm{PPF}}(s)=\frac{-1.24 \times 10^{16}}{4.789 \times 10^{7} s^{3}+0.01656 s^{2}+3.591 \times 10^{5} s} .
$$

Substituting the values of $a, \alpha, C_{p}$, and $\omega_{c}$ into (12) shows that the impedance is active.

The location of the closed-loop poles are shown in Fig. 7. By comparing the locations of the closed-loop poles of the active piezoelectric shunt controller, shown in Fig. 7, and the passive controller, shown in Fig. 5, it can be clearly observed that the active controller is capable of reducing the effective cantilever $Q$ factor much more than the passive controller.

Note that, it would be possible to almost completely damp the first mode of the cantilever with a PPF controller [18] for

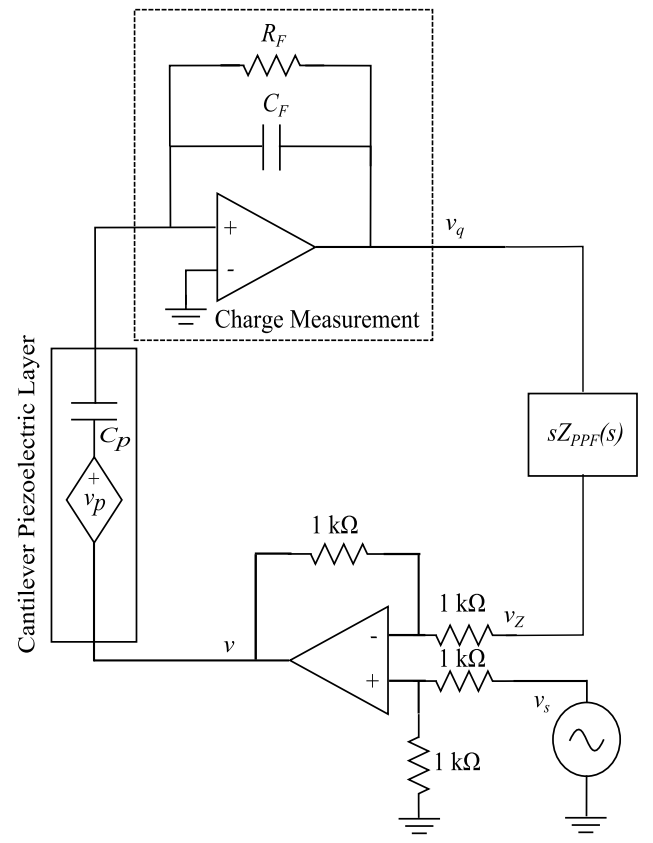

Fig. 8. Implementation of a synthetic impedance.

such applications where the cantilever is being operated at the second resonance. When scanning on the first resonance mode this amount of damping would mean that the force sensitivity would be reduced significantly and it would not be possible to obtain an image.

\section{ImPlementation OF THE ACTIVE IMPEDANCE}

When the desired impedance transfer function is active, the impedance cannot be built from passive components. To overcome this issue, it is possible to build a synthetic impedance from active components.

A passive impedance $Z(s)=L s+R$ was implemented synthetically in [11] by measuring the terminal voltage $v_{z}(s)$ and controlling the terminal current $i_{z}(s)$ according to the relationship $i_{z}(s)=v_{z}(s) / Z(s)$. The voltage to current relationship will therefore mimic the desired impedance. In this implementation, the voltage $v_{z}$ was measured then passed through a filter $Y(s)=1 / Z(s)$. The voltage output of $Y(s)$ was then converted to a current $i_{z}$ with a voltage-controlled current source.

The derived shunt impedance required to implement the PPF controller is strictly proper. Hence, the corresponding admittance is not a proper transfer function. Therefore, the implementation of this impedance should be done by measuring the terminal current $i_{z}(s)$ and controlling the terminal voltage $v_{z}(s)$ according to the relationship $v_{z}(s)=i_{z}(s) Z(s)$. The main issue with current measurement is measurement noise. The current is measured with a transimpedance amplifier in series with the internal capacitance of the piezoelectric transducer resulting in a differentiator, which amplifies high frequency noise. To avoid this problem, charge is measured and the impedance is implemented according to the relationship $v_{z}(s)=q_{z}(s) s Z(s)$. The synthetic impedance circuit designed to implement this, using Linear Technology LT1468 


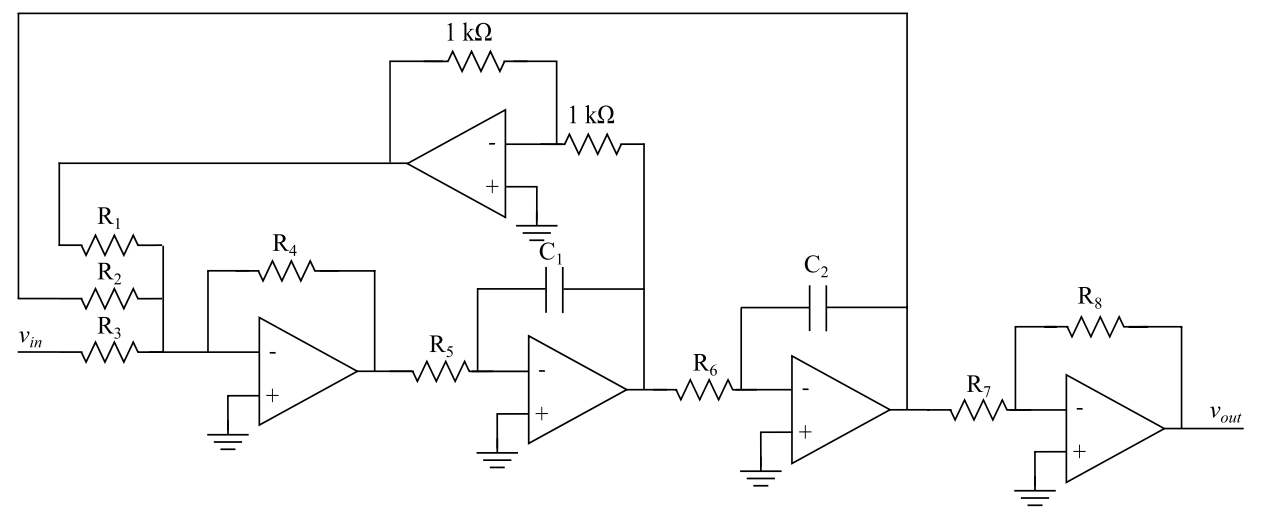

Fig. 9. State variable filter designed to implement $s Z_{\mathrm{PPF}}(s)$.

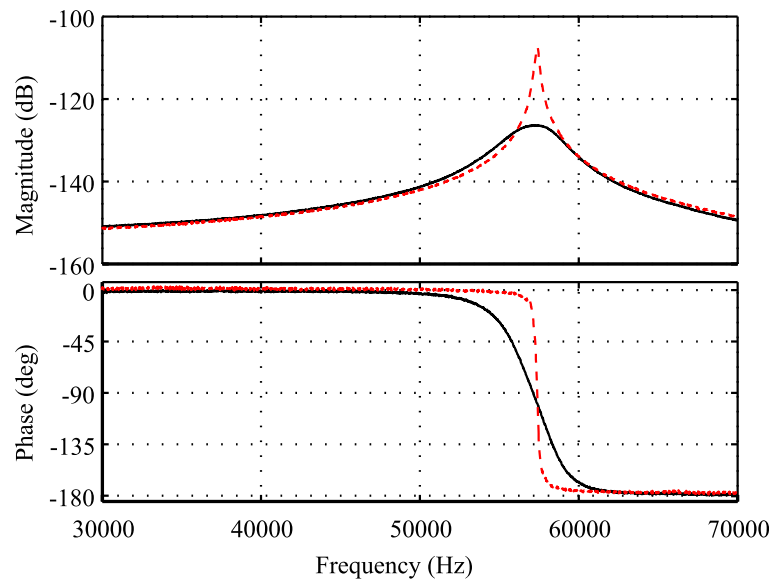

Fig. 10. Frequency response of $G_{d v_{s}}(s)$ with the cantilever in open loop $(--)$ and $H(s)^{-1} G_{d v_{s}}(s)$ with the cantilever in closed loop (-).

operational amplifiers [27], is shown in Fig. 8. The required filter is now $s Z(s)$ rather than $Z(s)$. Multiplying (10) by $s$ results in the transfer function

$$
s Z_{\mathrm{PPF}}(s)=\frac{a}{\alpha C_{p} s^{2}+2 \alpha C_{p} \zeta_{c} \omega_{c} s+\alpha C_{p} \omega_{c}^{2}-a C_{p}}
$$

which was implemented with a state variable filter using operational amplifiers, as shown in Fig. 9. Note that, it would be possible to design $s Z_{\mathrm{PPF}}(s)$ with a much simpler design such as the Sallen-Key filter topology. However, the state variable design has the advantage over the Sallen-Key design in that it has improved stability and adjustment of parameters is easier. With the Sallen-Key filter design, it is not possible to independently tune the filter resonance frequency and $Q$ factor. The transfer function from the input voltage to the output voltage of the state variable filter is

$$
\frac{v_{\text {out }}}{v_{\text {in }}}=\frac{\frac{R_{4}^{3}}{R_{1} R_{2} R_{3} R_{5} R_{6} C_{1} C_{2}}}{s^{2}+\frac{R_{4}}{R_{1} R_{5} C_{1}} s+\frac{R_{4}^{2}}{R_{1} R_{2} R_{5} R_{6} C_{1} C_{2}}} .
$$

The charge measurement block of the circuit shown in Fig. 8 produces a voltage $v_{q}$, which is proportional to the charge on the terminals of the cantilever piezoelectric layer. Ideally, without the resistor $R_{F}$ in parallel to the feedback capacitor,

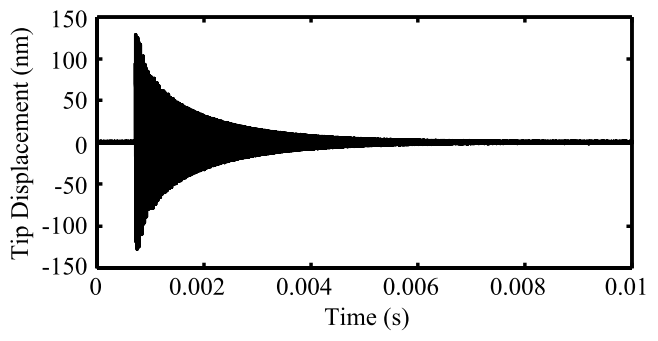

Fig. 11. Step response $G_{d v_{s}}(s)$ with no active shunt control applied.

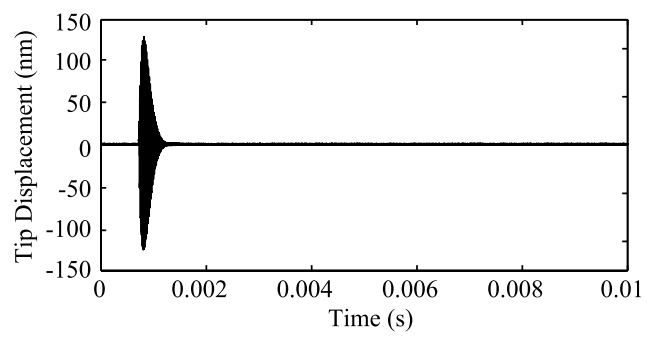

Fig. 12. Step response of $G_{d v_{s}}(s)$ with active shunt control applied.

the transfer function from $v$ to $v_{q}$ is

$$
\frac{v_{q}}{v}=\frac{C_{p}}{C_{F}} .
$$

As charge is the integral of current, the slightest offset in current leads to a saturation of the operational amplifier over time. To avoid this behavior, the resistor $R_{F}$ is placed in parallel to $C_{F}$. The transfer function from $v$ to $v_{q}$ is now

$$
\frac{v_{q}}{v}=\frac{R_{F} C_{p} s}{1+R_{F} C_{F} s} .
$$

This introduces a high-pass filter, which means that at low frequencies current is measured rather than charge. Note that the corner frequency of the high-pass filter has to be below the frequency of the first cantilever mode, otherwise the current and not the charge will be measured in the relevant frequency range.

$R_{F}$ was set to a value of $120 \mathrm{k} \Omega$ and $C_{F}$ set to a value of $0.82 \mathrm{nF}$. These values result in a cutoff frequency for the highpass filter of $1617 \mathrm{~Hz}$, which ensures that charge is measured 


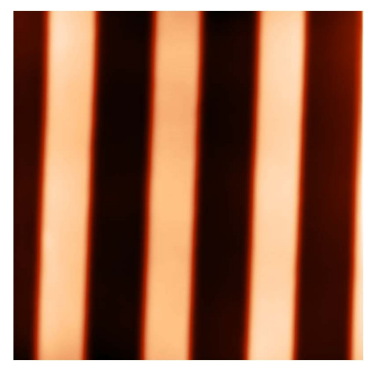

(a)

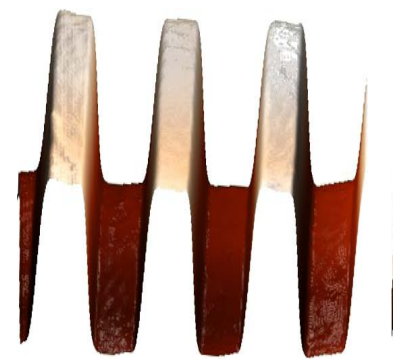

(d)

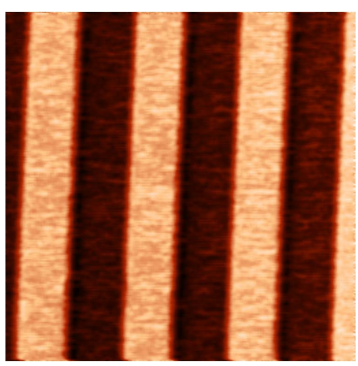

(b)

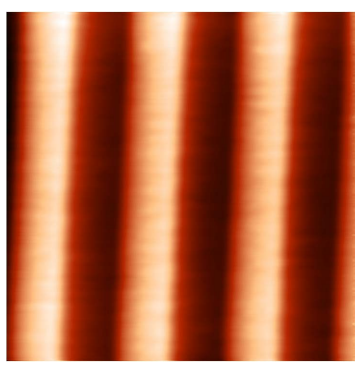

(c)

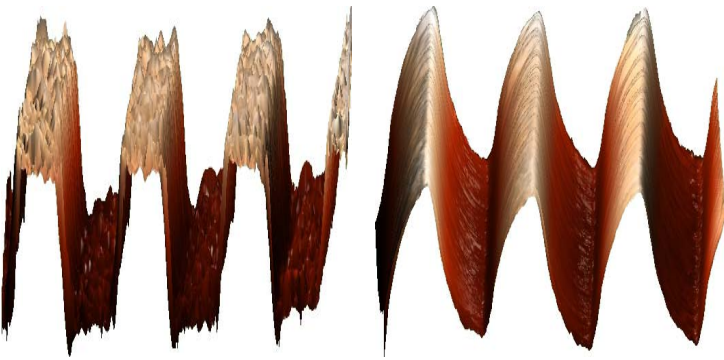

(e)

(f)

Fig. 13. Images of the NT-MDT TGZ1 calibration grating obtained at a scan speed of $60 \mu \mathrm{m} / \mathrm{s}$. The maximum value of $K_{Z}$, which ensured loop stability, was used with the active shunt connected. When the shunt is removed the $z$-axis feedback loop becomes unstable. $K_{Z}$ must be reduced by a factor of 7 for stability to be regained. (a) 2-D image with active shunt control. (b) 2-D image with no $Q$ control. (c) 2-D image with no $Q$ control. $K_{Z}$ reduced by a factor of 7. (d) 3-D image with active shunt control. (e) 3-D image with no $Q$ control. (f) 3-D image with no $Q$ control. $K_{Z}$ reduced by a factor of 7 .

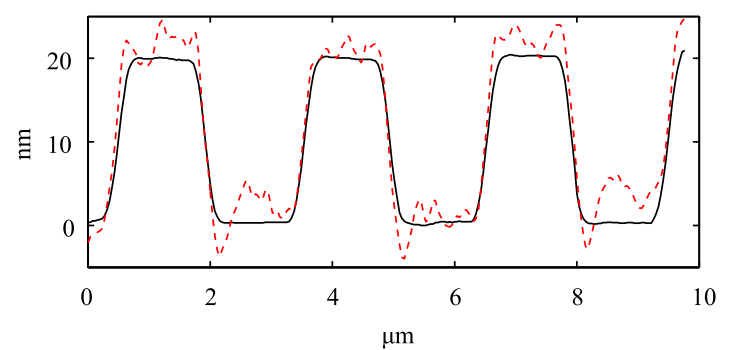

Fig. 14. Cross section of the NT-MDT TGZ1 sample topography obtained with (-) and without (- -) active piezoelectric shunt control. In both images, the $z$-axis feedback is set at the same value.

rather than current at the cantilevers resonance frequency of $57406 \mathrm{kHz}$.

\section{EXPERIMENTAL RESULTS}

\section{A. Measurement of Cantilever $Q$ Factor}

To demonstrate the effectiveness of the active piezoelectric shunt controller, the frequency response of $H^{-1}(s) G_{d v_{s}}(s)$ was measured rather than $G_{d w}(s)$ due to the difficulties in measuring $G_{d w}(s)$. To measure the frequency response of $G_{d w}(s)$, a separate piezoelectric actuator must be placed under the cantilever to excite it. The difficulty with this is that the cantilever mounting adds additional dynamics to the system and it is hard to find an actuator with resonance modes higher than those of the DMASP microcantilever.

$H^{-1}(s) G_{d v_{s}}(s)$ has the same poles as $G_{d w}(s)$ therefore measuring the frequency response of $H^{-1}(s) G_{d v_{s}}(s)$ is sufficient for comparing the damping of the open- and closed-loop system.

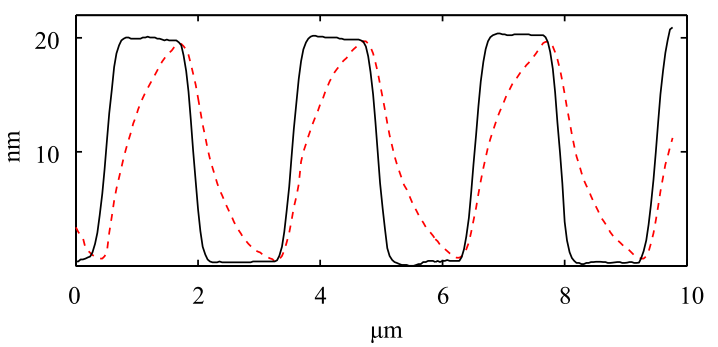

Fig. 15. Cross section of the NT-MDT TGZ1 sample topography obtained with (-) and without (- -) active piezoelectric shunt control. To obtain the image without shunt control the $z$-axis feedback was reduced by a factor of 7 .

To obtain $H^{-1}(s) G_{d v_{s}}(s), H(s)$ was first obtained by applying a pseudorandom signal to $v_{s}$ and measuring the frequency response across the terminals of the piezoelectric transducer on the cantilever. $G_{d v_{s}}(s)$ was then measured by applying a pseudo random signal to $v_{s}$ and measuring the cantilever displacement with the Polytec MSV 400. $H^{-1}(s) G_{d v_{s}}(s)$ was then obtained offline in MATLAB, as shown in Fig. 10. From the frequency response, a significant reduction in the cantilever $Q$ factor can be observed.

The effective cantilever $Q$ factor may be determined from its step response. When the cantilever is approximated as a second-order system, the exponential decay rate of its step response is $\sigma=\omega_{n} / 2 Q . \omega_{n}$ is measured from the frequency response of Fig. 10 and $\sigma$ is measured from the step responses of Figs. 11 and 12. Note that, the step response for the piezoelectric shunt controlled system is for $G_{d v_{s}}(s)$, which includes the prefilter $H(s)$. The poles of $H(s)$ have a real part of -17300 , which places the poles of $H(s)$ much deeper 
into the left half of the complex plane than the poles of $H^{-1}(s) G_{d v_{s}}(s) . H(s)$ will therefore have little effect on the step response. A reduction in the effective $Q$ factor from 226 in open loop to 17 in closed loop was observed.

\section{B. AFM Imaging Performance}

To demonstrate the effectiveness of the active piezoelectric shunt control technique, an NT-MDT NTEGRA AFM [28] was fitted with the DMASP microcantilever. Images of a NT-MDT TGZ1 [28] calibration grating were obtained with and without the active piezoelectric shunt controller. The NT-MDT TGZ1 grating consists of a periodic step formed from silicon dioxide with a period of $3 \pm 0.05 \mu \mathrm{m}$ and step height of $18.5 \pm 1 \mathrm{~nm}$.

Scans were obtained on a $10 \mu \mathrm{m} \times 10 \mu \mathrm{m}$ section of the calibration grating at a scan speed of $60 \mu \mathrm{m} / \mathrm{s}$. Images were obtained with no $Q$ control $(Q=226)$ and with the active piezoelectric shunt controller applied to reduce the cantilever $Q$ factor to 17 . With the active piezoelectric shunt controller applied the $Z$ axis feedback controller gain $\left(K_{Z}\right)$ was increased until the feedback loop became unstable. $K_{Z}$ was then reduced slightly to ensure closed-loop stability. The 2- and 3-D images obtained are shown in Fig. 3(a) and (d). The active piezoelectric shunt controller was then removed to return the cantilever $Q$ factor to 226. The resulting 2-D and 3-D images obtained are shown in Fig. 3(b) and (d). A cross section of the scans in closed and open loop is shown in Fig. 14. The instability in the $z$-axis feedback loop can clearly be observed in the image obtained with the higher cantilever $Q$ factor. To remove the instability in the $z$-axis, feedback loop $K_{Z}$ must be reduced. $K_{Z}$ was reduced by a factor of 7 before the oscillations in the image disappeared, as shown in Fig. 13(c) and (f) and Fig. 15. It is clear from the images that reducing the cantilever $Q$ factor with active piezoelectric shunt control allows for improved image quality at high scan speeds.

\section{CONCLUSION}

This paper has demonstrated that the use of an active impedance in the piezoelectric shunt control framework allows for a further reduction in the cantilever $Q$ factor than is possible when a passive impedance is used. A PPF controller was designed, which resulted in significant damping of the cantilever. An active impedance was then designed such that the piezoelectric shunt control system would emulate this PPF controller. A significant reduction in the cantilever $Q$ factor was achieved using this technique. AFM images of a test sample demonstrate the improvement in image quality at high scan speeds obtainable when the active piezoelectric shunt controller is applied.

Passive piezoelectric shunt control has previously been used to damp the first resonant mode of the piezoelectric scanner, which moves the sample in the lateral axes. This was demonstrated in [29] and [30] with a piezoelectric tube scanner and in [31] with a piezoelectric stack actuated flexurebased scanner. Increasing the bandwidth of the scanner reduces the risk of exciting the scanner's resonance at high scan speeds. The technique of active piezoelectric shunt control may be applied to the AFM piezoelectric scanner to further increase the scanner bandwidth. This is the subject of future work.

\section{REFERENCES}

[1] G. Binnig, C. Gerber, E. Stoll, T. R. Albrecht, and C. F. Quate, "Atomic resolution with atomic force microscope," Europhys. Lett., vol. 3, no. 12, p. $1281,1987$.

[2] V. B. Elings and J. A. Gurley, "Jumping probe microscope," U.S. Patent 5266801 , Nov. 30, 1993.

[3] Q. Zhong, D. Inniss, K. Kjoller, and V. Elings, "Fractured polymer/silica fiber surface studied by tapping mode atomic force microscopy," Surf. Sci., vol. 290, nos. 1-2, pp. L688-L692, 1993.

[4] R. Garcia and R. Perez, "Dynamic atomic force microscopy methods," Surf. Sci. Rep., vol. 47, nos. 6-8, pp. 197-301, 2002.

[5] G. Borionetti, A. Bazzali, and R. Orizio, "Atomic force microscopy: A powerful tool for surface defect and morphology inspection in semiconductor industry," Eur. Phys. J. Appl. Phys., vol. 27, nos. 1-3, pp. 101-106, 2004.

[6] T. Ando, N. Kodera, Y. Naito, T. Kinoshita, K. Furuta, and Y. Y. Toyoshima, "A high-speed atomic force microscope for studying biological macromolecules in action," ChemPhysChem, vol. 4, no. 11, pp. 1196-1202, 2003.

[7] N. Kodera, D. Yamamoto, R. Ishikawa, and T. Ando, "Video imaging of walking myosin V by high-speed atomic force microscopy," Nature, vol. 468, no. 7320, pp. 72-76, 2010.

[8] T. Sulchek, R. Hsieh, J. D. Adams, G. G. Yaralioglu, S. C. Minne, C. F. Quate, et al., "High-speed tapping mode imaging with active Q control for atomic force microscopy," Appl. Phys. Lett., vol. 76, no. 11, pp. 1473-1475, 2000.

[9] T. Sulchek, G. G. Yaralioglu, C. F. Quate, and S. C. Minne, "Characterization and optimization of scan speed for tapping-mode atomic force microscopy," Rev. Sci. Instrum., vol. 73, no. 8, pp. 2928-2936, Aug. 2002.

[10] M. W. Fairbairn and S. O. R. Moheimani, "Resonant control of an atomic force microscope micro-cantilever for active Q control," Rev. Sci. Instrum., vol. 83, no. 8, pp. 083708-1-083708-9, Aug. 2012.

[11] M. W. Fairbairn, S. O. R. Moheimani, and A. J. Fleming, "Q-control of an atomic force microscope micro-cantilever: A sensor-less approach," IEEE/ASME J. Microelectromechan. Syst., vol. 20, no. 6, pp. 1372-1381, Dec. 2011.

[12] M. Balas, "Feedback control of flexible systems," IEEE Trans. Autom. Control, vol. 23, no. 4, pp. 673-679, Aug. 1978.

[13] R. Stark, "Time delay Q-control of the microcantilever in dynamic atomic force microscopy," in Proc. 5th IEEE Conf. Nanotechnol., vol. 1. Jul. 2005, pp. 259-262.

[14] D. R. Sahoo, T. De, and M. Salapaka, "Observer based imaging methods for atomic force microscopy," in Proc. 44th IEEE Conf. CDC ECC, Dec. 2005, pp. 1185-1190.

[15] M. W. Fairbairn and S. O. R. Moheimani, "Quality factor enhancement of an atomic force microscope micro-cantilever using piezoelectric shunt control," in Proc. IEEE/ASME Int. Conf. Adv. Intell. Mechatron., Kaosiung, Taiwan, Jul. 2012, pp. 556-561.

[16] G. Meyer and N. M. Amer, "Novel optical approach to atomic force microscopy," Appl. Phys. Lett., vol. 53, no. 12, pp. 1045-1047, 1988.

[17] R. Kassies, K. O. van der Werf, M. L. Bennink, and C. Otto, "Removing interference and optical feedback artifacts in atomic force microscopy measurements by application of high frequency laser current modulation," Rev. Sci. Instrum., vol. 75, no. 3, pp. 689-693, 2004.

[18] M. W. Ruppert, M. Fairbairn, and S. O. R. Moheimani, "Multi-mode resonant control of a micro-cantilever for atomic force microscopy," in Proc. IEEE/ASME Int. Conf. Adv. Intell. Mechatron., Wollongong, Australia, Jul. 2013, pp. 1-12.

[19] C. J. Goh and T. K. Caughey, "On the stability problem caused by finite actuator dynamics in the collocated control of large space structures," Int. J. Control, vol. 41, no. 3, pp. 787-802, 1985.

[20] J. J. Dosch, D. J. Inman, and E. Garcia, "A self-sensing piezoelectric actuator for collocated control," J. Intell. Mater. Syst. Struct., vol. 3, pp. 166-185, May 1992.

[21] H. R. Pota, S. O. R. Moheimani, and M. Smith, "Resonant controllers for smart structures," Smart Mater. Struct., vol. 11, no. 1, pp. 1-8, 2002. 
[22] A. J. Fleming and S. O. R. Moheimani, "Control oriented synthesis of high performance piezoelectric shunt impedances for structural vibration control," IEEE Trans. Control Syst. Technol., vol. 13, no. 1, pp. 98-112, Jan. 2005.

[23] S. O. R. Moheimani, A. J. Fleming, and S. Behrens, "On the feedback structure of wideband piezoelectric shunt damping systems," Smart Mater. Struct., vol. 12, no. 1, pp. 49-56, Feb. 2003.

[24] F. Kuo, Network Analysis and Synthesis. New York, NY, USA: Wiley, 1966.

[25] S. Moheimani and A. J. Fleming, Piezoelectric Transducers for Vibration Control and Damping. New York, NY, USA: Springer-Verlag, 2006.

[26] (2012). Bruker AFM Probes Website [Online]. Available: http://www.brukerafmprobes.com/p-3252-dmasp.aspx

[27] Linear Technology LT1468 Datasheet, Linear Technology Website, Camas, WA, USA, 2012.

[28] NT-MDT Atomic Force Microscopy, NT-MDT, Moscow, Russia, 2012.

[29] A. J. Fleming and S. O. R. Moheimani, "Sensorless vibration suppression and scan compensation for piezoelectric tube nanopositioners," IEEE Trans. Control Syst. Technol., vol. 14, no. 1, pp. 33-44, Jan. 2006.

[30] S. Aphale, A. J. Fleming, and S. O. R. Moheimani, "High speed nanoscale positioning using a piezoelectric tube actuator with active shunt control," Micro Nano Lett., vol. 2, no. 1, pp. 9-12, Jan. 2007.

[31] A. A. Eielsen and A. J. Fleming, "Passive shunt damping of a piezoelectric stack nanopositioner," in Proc. Amer. Control Conf., Baltimore, MD, USA, Jun. 2010, pp. 4963-4968.

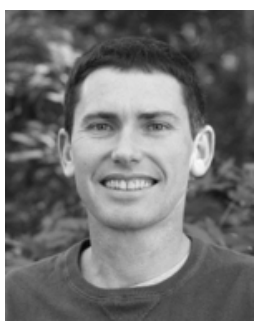

Matthew W. Fairbairn (M'10) received the bachelor's and Ph.D. degrees in electrical engineering from the University of Newcastle, Callaghan, Australia, in 2009 and 2013, respectively.

He was a Control Systems Engineer, from 2007 to 2009 , where he was developed the supervisory control and data acquisition systems for the water and waste water industry. He is currently a PostDoctoral Fellow with the Laboratory for Dynamics and Control of Nanosystems, University of Newcastle. His current research interests include the limitations of high-speed atomic force microscopy due to piezoelectric actuator and cantilever dynamics.

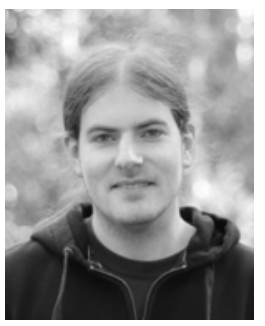

Philipp Müller received the bachelor's degree in mechatronics engineering from the University of Stuttgart, Stuttgart, Germany, in 2008.

$\mathrm{He}$ is currently with the University of Newcastle, Callaghan, Australia, where he is involved about the control of microcantilever dynamics for high-speed atomic force microscopy. His current research interests include systems theory and automatic control.

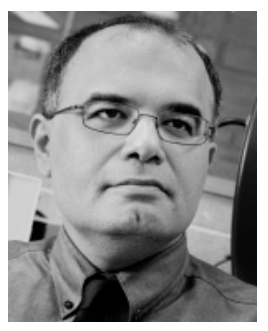

S. O. Reza Moheimani (F'11) received the B.Sc degree in electrical and electronic engineering from Shiraz University, Shiraz, Iran, and the M.Eng.Sc. and Ph.D. degrees in electrical and electronic engineering from the University of New South Wales, Sydney, Australia, and the Australian Defense Force Academy, Canberra, Australia, in 1990, 1993, and 1996, respectively.

He joined the University of Newcastle, Callaghan, Australia, in 1997, where he is a Professor of electrical engineering and an Australian Research Council Future Fellow. He is the Founder and Director with the Laboratory for Dynamics and Control of Nanosystems, University of Newcastle. His current research interests include ultrahigh-precision mechatronic systems, with particular emphasis on dynamics and control at the nanometer scale, including applications of control and estimation in nanopositioning systems for highspeed scanning-probe microscopy, modeling and control of microcantileverbased devices, control of microactuators in microelectromechanical systems, and control issues related to ultrahigh-density probe-based data-storage systems.

Dr. Moheimani is a fellow of the International Federation of Automatic Control (IFAC) and the Institute of Physics, London, U.K. He was a recipient of the IFAC Nathaniel B. Nichols Medal in 2014, the IFAC Mechatronic Systems Award in 2013, the IEEE Transactions on Control Systems Technology Outstanding Paper Award in 2007, and the IEEE Control Systems Society Control Systems Technology Award in 2009, with the latter shared with a group of researchers from IBM Research-Zurich, Zurich, Switzerland, where he has held several visiting appointments. 